\title{
MIGRACIONES, GÉNERO Y SUBJETIVIDAD: EL TRABAJO CON POBLACIÓN MIGRANTE EN COSTA RICA
}

\section{MIGRATIONS, GENDER AND SUBJECTIVITY: WORKING WITH MIGRANT POPULATION IN COSTA RICA}

\author{
Silvia Azofeifa Ramos* \\ Carmen Caamaño Morúa** \\ Anna Matteucci Wo Ching***
}

RESUMEN

\begin{abstract}
El siguiente artículo es un análisis de las primeras experiencias de trabajo del Seminario de Extensión Docente "Migración y Subjetividad. Trabajando con población migrante", el cual se propone como un espacio para reflexionar sobre transformaciones subjetivas en el contexto de las migraciones. Estas implican una imbricación entre lo material, la construcción cultural y los procesos inter e intrasubjetivos, tanto de la población migrante como de los y las profesionales que trabajan con esta.
\end{abstract}

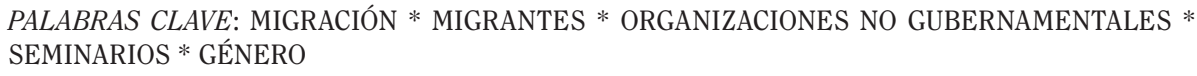

\section{ABSTRACT}

The following article is an analysis of the first experiences working Extension Teaching Seminar "Migration and Subjectivity. Working with migrants", which is proposed as a space to reflect on subjective transformations in the context of migration, which involve an overlap between the material, cultural construction and inter-and intra-subjective processes of both the migrant population as of the professionals who work with it. KEYWORDS: MIGRATION * MIGRANTS * NONGOVERNMENTAL ORGANIZATION * SEMINARS *
GENDER

Instituto de Investigaciones Sociales (IIS) de la Universidad de Costa Rica (UCR).

silvia.azofeifa@gmail.com

** Instituto de Investigaciones Sociales (IIS) de la Universidad de Costa Rica (UCR). carmen.caamano@ucr.ac.cr

*** Instituto de Investigaciones Sociales (IIS) de la Universidad de Costa Rica (UCR). anna.matteucci@ucr.ac.cr 


\section{INTRODUCCIÓN}

El Seminario de Extensión Docente "Migración y Subjetividad. Trabajando con población migrante"l es una nueva aproximación de la investigación con personas que trabajan con población migrante en diversos contextos. Los contenidos del Seminario forman parte de algunos de los resultados de la investigación sobre comunidades transnacionales desarrollada por Caamaño desde el año 2005 (Caamaño, 2010a, 2010b, 2010c, 2011, 2012a y 2012b), en la que participaron Yirlanny Navarro y Jazmín Agüero. Actualmente, participan Anna Matteucci Wo Ching, incorporada a la investigación desde el 2009 (Caamaño, Navarro, Matteucci y Agüero, 2010; Matteucci, 2011) y Silvia Azofeifa Ramos desde el 2011.

En este trabajo se integra una perspectiva teórico-metodológica a partir de un modelo de reflexión-afecto-reflexión-acción, el cual sigue las enseñanzas de Paulo Freire (1983) y Fals Borda (1986) sobre reflexión-acción, pero incorpora el afecto como un elemento fundamental para desarrollar el conocimiento.

El afecto es usualmente excluido del plano reflexivo, por lo tanto, para incluirlo se utiliza una metodología basada en actividades lúdicas, artísticas y juego de roles. El modelo de reflexión-afecto-reflexión-acción se inserta a su vez, dentro de la perspectiva denominada Economía Política Cultural Crítica. En esta se integran los procesos de acumulación capitalista con procesos de construcción cultural y subjetiva para comprender la realidad (Caamaño, 2011).

El Seminario de Extensión Docente se encuentra dirigido a personas que trabajan con población migrante y contó con una participación mayoritaria de mujeres, de las cuales muchas realizan labores de tipo asistencial en organizaciones dirigidas a la población migrante. Respecto a la asistencia al Seminario, participaron aproximadamente 30 personas divididas en grupos $y$ de estas, 3 fueron hombres.

$\overline{1}$ El equipo de trabajo se encuentra conformado por Carmen Caamaño, Anna Matteucci y Silvia Azofeifa.
La reflexión sobre el Seminario está fundamentada en tres ejes entrelazados de discusión: las migraciones, la subjetividad y el género. A partir de estos, se trabajan temas tales como: las mujeres en la provisión de cuidados, el trabajo reproductivo, las cadenas globales del cuido y el lugar que ocupan las trabajadoras de Organizaciones No Gubernamentales (ongs) y personas voluntarias que atienden a la población migrante, en la cadena de cuidados generada en el capitalismo neoliberal.

Por ende, este artículo busca reflexionar sobre el trabajo con las personas participantes en los dos primeros grupos del Seminario $y$ al mismo tiempo, se convierte en una forma de continuar el diálogo con ellas y con quienes actualmente participan en este.

\section{LAS MIGRACIONES}

La finalidad del Seminario de Extensión Docente "Migración y Subjetividad: Trabajando con población Migrante" es intercambiar nuestra perspectiva sobre migraciones transnacionales con personas que trabajan el tema migratorio en Costa Rica. Se pretende compartir la idea de que las poblaciones migrantes no son solo números que aumentan las estadísticas en un país y las disminuyen en otros, sino seres humanos con subjetividades en constante construcción que establecen vínculos entre geografías y culturas a través de fronteras; que no se mueven solos o solas, sino que llevan consigo una historia, vínculos con lugares y personas en diferentes espacios geográficos y una serie de expectativas con sus respectivos afectos.

Para analizar la experiencia migratoria en un marco intersubjetivo, se desarrollaron diferentes técnicas lúdicas y artísticas, en las cuales cada persona aporta al conocimiento según su propia experiencia. De esta manera, durante la primera y última sesión, los participantes jugaron "Entre aquí y allá: el juego de la migración" (Caamaño, Navarro, Matteucci y Agüero, 2010), el cual intenta recrear en el plano simbólico, la concepción de espacio transnacional, entendido como campos sociales contradictorios y ambiguos, que involucran tanto intercambios materiales entre personas $y$ grupos situados en lugares geográficos $y$ 
culturalmente distantes, como intercambios y construcciones simbólicas significativas afectivamente (Caamaño, 2010b, 2010c y 2011).

Se parte del espacio transnacional como un espacio psíquico transicional (Winnicott, citado en Caamaño, 2010b y 2011) en donde es necesario generar un "espacio suficientemente bueno"2 para que las personas puedan vivir las migraciones, así como, las dificultades que estas puedan traer con la finalidad de fortalecerse en el proceso. Generar este espacio implica la posibilidad de mantener la comunicación constante entre aquellos que se van $y$ aquellos que se quedan. Sin embargo, las personas participantes en los dos seminarios del primer semestre de 2012, mostraron cómo no todas las personas pueden lograr esto. El caso más claro es el de aquellas con la condición de refugiadas, quienes no pueden comunicarse con su familia y seres queridos en el lugar de origen, ya que de ser rastreadas pueden correr peligro.

Además, para generar un "espacio suficientemente bueno" es necesario que en el país al que llegan las personas migrantes, exista el respeto a los derechos humanos, así como, condiciones adecuadas como: el acceso a la salud $y$ a la educación, entre otras; situación que no siempre sucede, como es el caso de la mayoría de la población nicaragüense que inmigra a Costa Rica. Evidenciar la diferencia en el trato hacia inmigrantes nicaragüenses y población refugiada permite cuestionar también las exclusiones que se ejercen en contra de algunas poblaciones nacionales, como por ejemplo, la población indígena, la cual también sufre de discriminación, exclusión social y una constante violación de sus derechos.

Durante las discusiones en el Seminario, se observó cómo la clase social, la etnia, la nacionalidad, la religión, el género, entre otras, son categorías a partir de las cuales se construyen "otredades", y cómo la creación de otredad

$2 \quad$ Según Winnicott, el espacio transicional debe proporcionar condiciones básicas de seguridad $y$ protección para que los niños puedan enfrentarse con lo nuevo en sus vidas y aprehender la realidad. Estas condiciones deben estar presentes también en el espacio transnacional construido por la población migrante (Winnicott, citado en Caamaño, 2010b y 2011). obstaculiza la posibilidad de crear comunidad. La nueva Ley de Migración y Extranjería nro. 8764 plantea la búsqueda de integración y el respeto a los derechos humanos; no obstante, según participantes del Seminario, en realidad dicha ley lo que hace es excluir un sector importante de población inmigrante, estableciendo barreras como costos y papeleos, los cuales obstaculizan su incorporación.

Ante las limitaciones que se presentan a las poblaciones inmigrantes a nivel gubernamental, surgen iniciativas por parte de organizaciones no gubernamentales, religiosas $y$ comunitarias para atender las necesidades más inmediatas de estas personas.

En los dos primeros grupos del Seminario impartido, los participantes corresponden en su mayoría a mujeres, las cuales atienden a migrantes económicos ${ }^{3} y$ a población refugiada desde organizaciones no gubernamentales o religiosas. Además, participaron personas con experiencia migratoria: migrantes nicaragüenses, estadounidenses, refugiadas, personas que han buscado opciones de trabajo o estudio fuera del país o en otra región de Costa Rica. En algunos casos, la migración interna desde la zona rural a la zona urbana central fue reconocida como una vivencia igualmente importante e impactante que la migración internacional. También, asistieron personas con familiares que han sido o son inmigrantes económicos en Estados Unidos, por lo que se encuentra que quienes atienden a la población inmigrante, de una $u$ otra manera, viven o han vivido personalmente procesos de migración.

Esta experiencia subjetiva permite que al hablar sobre migración en el Seminario, se enfatice en la diversidad de experiencias, pues no es igual la emigración de diplomáticos o de personas que salen a estudiar, que la vivida por migrantes económicos indocumentados, mujeres migrantes, personas en condición de refugio $o$ la experiencia de niños $y$ adolescentes.

Asimismo se discute la migración como opción de vida para quienes el lugar de origen

$3 \quad$ Estas son aquellas personas que migran atendiendo necesidades económicas, que buscan trabajo en otros países con el fin de garantizar su supervivencia y la de sus familias. 
no representa el lugar deseado para vivir y ser feliz. En el primer grupo del Seminario se plantean las redes de apoyo para las personas migrantes, como un tejido que no necesariamente involucra a la familia. Por otro lado, para el segundo grupo, la familia es la red más importante de la que se espera apoyo y comprensión durante los procesos de migración y la vida en general.

Cuando se analiza la migración a través de la lente del género, la decisión de emigrar se vincula no solo a las condiciones económicas en el lugar de origen, sino que se suman aspectos contextuales de discriminación y violencia basada en género, lo cual puede persistir aun en el lugar de destino.

\section{SUBJETIVIDADES Y AFECTOS}

En el Seminario se abordó el tema de la subjetividad dentro de la labor realizada con población migrante, tanto de quienes migran como de quienes se desempeñan en las diversas instituciones. Dentro de las discusiones grupales sobre subjetividad y migraciones, emergió de manera significativa el tema de los afectos, especialmente, los vinculados con duelos generados a partir de la separación, lo cual implica dejar físicamente la familia, las amistades, la comunidad y el país de origen.

Se puede hablar de una mediación cognitiva, que facilita o dificulta a las personas migrantes, sus familiares $y$ redes de apoyo, el manejo de los afectos que surgen en el proceso migratorio y la manera en que dichos afectos son explicados teóricamente en la literatura sobre migraciones, enfocándose en la separación y el duelo, los cuales producen depresión (Caamaño, 2010b y 2011). Dicha mediación se manifiesta en el modo en que las personas que atienden y trabajan con la población migrante, se relacionan con esta, ya sea con afectos de identificación o de rechazo. Sin embargo, es poco común que se reflexione críticamente sobre el tema de los afectos en la práctica cotidiana de las organizaciones.

El punto de partida para entender la identificación de las personas participantes del Seminario con el tema de las migraciones, es su vivencia personal con la migración. Por el origen extranjero de muchas personas participantes, se evidenció en las reflexiones cómo ellos y ellas mismas han atravesado muchos de los trámites, así como, los obstáculos burocráticos y emocionales que la mayoría viven antes, durante $y$ después de su ingreso al país. En el caso de participantes que emigraron de zonas rurales a zonas urbanas, en búsqueda de estudio y oportunidades laborales, se habló de sentimientos de extrañamiento $y$ algunas experiencias marcadas por estereotipos que se tienen de sus provincias o regiones de origen. De la experiencia personal de haber vivido la emigración de familiares $y$ amistades, algunas de las personas asistentes expresan afectos relacionados con el duelo y el resentimiento, muchas veces vinculados a momentos de la vida en que había que cuidar de una persona enferma o de un niño, necesitando del apoyo de la persona que emigró.

Si bien, la experiencia migratoria de quienes atienden a población migrante puede ser de gran ayuda por la familiarización del personal con los diferentes procesos, las mismas funcionarias de organismos que trabajan con población migrante que participaron en el seminario, señalan que se debe prestar mucha atención para no confundir la experiencia de esa persona que atienden, con la vivida en la vida personal, ya que es una línea muy fácil de cruzar. La solidaridad y el cuido que esta identificación propicia es positiva, pero en algunas ocasiones puede entorpecer el objetivo principal de ayudar. Como asegura una de las participantes, poco se puede ayudar sentándose a llorar con la persona que se atiende.

En este sentido, afectos como el enojo, la ausencia de un ser querido o el resentimiento pueden movilizar a las personas que atienden a la población migrante, identificando su caso personal con el caso atendido y por ende, marcar el tipo de atención que se le brinda.

En el Seminario, algunas personas participantes advierten sobre la necesidad de reconocer los afectos propios y separarlos en su desempeño profesional. Formas particulares de interpretar la distancia y la separación producidas por las migraciones, además de concepciones tradicionales sobre la familia como institución social, generan vínculos 
específicos con la población migrante desde el trabajo institucional.

Otro aspecto de la subjetividad y las migraciones que se discutió en ambos grupos, fue el estilo de trabajo interinstitucional en el cual, la agilidad con la que diversos trámites se realizan, depende de una manera importante de las relaciones personales entre funcionarios $y$ funcionarias o personas encargadas. Así, la atención a la población migrante se ve permeada por la relación subjetiva, ya sea de cercanía y cordialidad, o de desconfianza y mala disposición entre diferentes actores de organizaciones vinculadas, prevaleciendo estas relaciones sobre las vías establecidas institucionalmente.

A partir de esta discusión, participantes del Seminario expresaron que al descansar sobre este tipo de favores y relaciones afectivas, se prescinde de la creación y sistematización de protocolos para la atención de personas migrantes, lo que conlleva a que se releguen trámites a persona particulares. De ahí que se observen funcionarios o funcionarias que se apropian, consciente o inconscientemente, de todo lo relacionado con casos y proyectos, asumiéndolos como propios e individuales, en lugar de responder a servicios que deben realizarse institucionalmente. Si bien, en los grupos del seminario se acepta la necesidad de definir pasos y procesos en la atención a migrantes, algunas personas participantes objetan que tampoco se puede estandarizar la atención como si las necesidades fueran las mismas para todas las personas, recalcando la diversidad dentro de las migraciones.

En relación con los afectos en la práctica laboral con población migrante, otro factor identificado fue la contención emocional ${ }^{4}$ ejercida por el personal en general, aun cuando no siempre han tenido capacitación para llevarla a cabo. En esta situación, el género tiene un papel importante, pues se espera que esa contención afectiva sea brindada por funcionarias

4 La contención emocional es la labor de apoyo que se realiza desde la Psicología para la atención de la tristeza, depresión, enojo, frustración u otros sentimientos manifestados por las personas migrantes, que corresponde a una concepción teórica, una metodología y procedimientos específicos que orientan la acción de quien la realiza. mujeres, siguiendo con los roles y las tareas de cuido atribuidas socialmente a ellas. En el segundo grupo del Seminario, está el caso de una mujer que trabaja como recepcionista en una organización para atención a migrantes, que en algunas oportunidades brinda por iniciativa propia, apoyo afectivo, contención y recomendaciones de tipo legal. En el mismo grupo, participó un hombre, para quién la carga emocional de escuchar los casos de las personas migrantes, muchas veces le generaba un sentimiento de impotencia y angustia difíciles de manejar, por lo que trataba de no tener vínculos emocionales con las personas que llegan a su organización.

Cabe rescatar que una gran parte de la ayuda social que se brinda a la población migrante, proviene de entidades cuyos servicios responden a la religión y la espiritualidad. Sin embargo, aunque es lógico esperar que los y las profesionales que atienden en estos centros religiosos profesen creencias afines, como señala una participante, es primordial que se haga una distinción clara entre su servicio y asesoría, así como, la caridad o asistencialismo de orden religioso. Para dicha participante, el trabajo que parte del asistencialismo, aunque puede ser bien intencionado, es susceptible de crear una relación de dependencia con las personas migrantes que son atendidas, generando así un considerable costo afectivo para ambas partes.

El hecho de que muchas de estas ongs sean religiosas, podría ser una limitante para plantear un abordaje distinto a la caridad $y$ asistencialismo en el trabajo con la población migrante. Cabe rescatar que estas organizaciones "están inmersas en un campo contradictorio de relaciones de poder definido por tres fuerzas contendientes: los estados nacionales, los movimientos religiosos fundamentalistas y los centros globales que gestionan la agenda neoliberal" (Brenner, s.f., citado en Caamaño, 2012).

El trabajo voluntario es otro de los componentes de análisis propiciado por el Seminario. Ante la falta de presupuesto, no se contrata suficiente personal en las organizaciones; por tanto, muchas personas que trabajan con población migrante se ven obligadas a asumir una carga de trabajo extenuante, fuera de horas 
laborales y sin remuneración, que se sostiene en la mayoría de los casos por los afectos e identificaciones dirigidas hacia la población migrante, entremezclándose la subjetividad con el compromiso profesional y ético.

\section{GÉNERO Y MIGRACIÓN: PROVISIÓN DE CUIDADOS EN CARENCIA DE ATENCIONES}

En la vivencia de lo afectivo y la atención directa a la población migrante, la experiencia con los dos primeros grupos del Seminario devela que hay un factor de construcción de género en sus experiencias personales con la migración, así como con sus experiencias en la atención de migrantes en instituciones, las cuales se enfocan desde lo judicial a lo educativo $y$ religioso, entre otras.

En Latinoamérica, persisten los papeles socialmente asignados a las mujeres en el ámbito familiar, la insuficiencia de los servicios para atender las necesidades familiares básicas, la falta de reconocimiento de la maternidad como responsabilidad social, así como prácticas relacionadas de la vida cotidiana, basadas en la concepción patriarcal de la familia (Montaño, 2010). A pesar de que existen avances en la incorporación de las mujeres al ámbito educativo y laboral, el Observatorio de Igualdad de Género de América Latina $y$ el Caribe constata que las mujeres no han alcanzado la igualdad ni la autonomía, en gran medida, porque no se quebró el cimiento básico de la desigualdad, el cual corresponde a la división sexual del trabajo y la consiguiente desnaturalización del trabajo doméstico como responsabilidad femenina (Montaño, 2010). Así, la provisión de cuidados se ha adjudicado casi exclusivamente a las mujeres, recargando sus responsabilidades, limitando su desarrollo personal y colocándolas en desventaja respecto a los hombres en el trabajo, estudio, recreación, participación política y atención a su salud, concatenándose así otras formas de exclusión y desigualdad (Carcedo, Chaves y Lexartza, 2011).

Al no asumirse el cuidado como responsabilidad social compartida, se generan grandes flujos migratorios de mujeres que buscan mejores condiciones para proveer cuidados a sus familias en el país de origen. Los mismos gobiernos fomentan la emigración de sus habitantes como medio para obtener remesas y como válvula de escape para el disenso político, aprovechándose de la diferencia salarial entre el país de origen y el de acogida (Caamaño, 2012b).

Entonces se constituyen "cadenas globales de cuido", en las que mujeres inmigrantes cuidan las familias de sus empleadoras, mientras que parte del dinero que obtienen en el extranjero es enviado a sus hogares de origen para el cuido de sus familias extendidas (Hondgneu-Sotelo y Ávila, 2006; Lan, 2006 citadas en Caamaño, 2012b). Para Carcedo, Chaves y Laxartza, lo que caracteriza estas cadenas globales es su carácter transnacional, el cual "involucra hogares de diferentes países y culturas, todos ellos con necesidades de cuidados particulares y que requieren establecer arreglos concretos de cuido que usualmente involucran diferentes tipos de recursos" (2011: 27).

Paradójicamente, la carencia de servicios públicos que las personas emigrantes enfrentan en sus países de origen, la encuentran en los países de acogida ante la limitación para que trabajadoras y trabajadores inmigrantes accedan a estos, ya que el trabajo de cuidado por parte de la población migrante "es usualmente el más precario, $y$ a la discriminación laboral que lo caracteriza, se suma la discriminación por origen nacional y condición migratoria" (Carcedo, Chaves y Lexartza, 2011). Las relaciones que se establecen en estas condiciones están marcadas por el poder particular $y$ desigual.

Esto ha ido creando la necesidad de generar una serie de servicios de apoyo para la población trabajadora inmigrante. Existen organizaciones religiosas, laicas y voluntarias, como también existen organizaciones feministas que luchan por los derechos de las mujeres trabajadoras inmigrantes. Estas últimas contrastan claramente con la labor asistencial de las anteriores; sin embargo, entre unas y otras se desarrollan lazos de solidaridad y lucha conjunta frente a acciones del Estado en contra de los derechos de la población inmigrante (Caamaño, 2012b).

El tema del cuidado y quién lo ejerce (para sí o para otros) remite a un problema de ejercicio de derechos, destacando la importancia de considerar el cuido como una obligación 
que se desprende del derecho al cuidado, comprendido como universal para todos los ciudadanos y no solo para las mujeres, el cual debe estar garantizado por los Estados en condiciones de igualdad (Pautassi, 2007).

En este seminario, al ser la mayoría de las participantes mujeres, surge la reflexión sobre temas como la migración vinculada con la violencia de género, así como el cuerpo como lugar de control social. Además, la conformación femenina de los grupos que participaron en el Seminario, sugiere la necesidad de profundizar en las estructuras que permean el trabajo realizado por ellas mismas, como mujeres en organizaciones no gubernamentales, educativas y religiosas en la atención de personas migrantes.

El plano doméstico familiar se reproduce en el ámbito laboral de estas funcionarias o voluntarias: "los cuidados no se asumen realmente como sociales, sino como responsabilidad particular de las personas y las familias, que deben satisfacer sus necesidades en este campo de acuerdo a los recursos de que disponen" (Carcedo, Chaves y Lexartza, 2011: 24).

Al igual que en la familia, en el espacio social, el cuido se convierte en un trabajo invisible, poco valorado y mal remunerado. Durante el desarrollo del Seminario, se observó cómo el trabajo del cuidado está incorporado en la subjetividad de las mujeres participantes, sin cuestionamiento al mandato social que recae sobre ellas ante la división sexual del trabajo, la desatención del Estado a las necesidades de cuido, en general y a las de la población migrante, en particular. En algunas organizaciones se reproduce una "ética reaccionaria de los cuidados", la cual de acuerdo con Carcedo, Chaves y Lexartza, tiene que ver con "una ética basada en la renuncia y el sacrificio, lo cual construye desigualdades entre quienes cuidan (mujeres) y quienes no cuidan. Lo anterior se impone por la vía del chantaje emocional, abriendo la posibilidad del abuso y la violencia en las relaciones destinadas a lo opuesto, el cuido" (2011: 28).

Ejemplo de lo anterior, es el cortometraje "El tren de las moscas" (2010), el cual muestra a un grupo de 14 mujeres de la comunidad de Guadalupe o La Patrona, en el Estado de
Veracruz (México), que desde hace 15 años dan comida y bebida a migrantes que viajan subidos en trenes de carga dirigidos hacia los Estados Unidos. Las personas migrantes las llaman "las patronas" por la localidad en la que se ubican, pero su referencia a la imagen religiosa de la Virgen de Guadalupe remite de nuevo a la idea de caridad y sacrificio que se establece desde lo religioso, como se puede ver en el hecho de que algunas patronas identifican a los migrantes con la figura en sufrimiento de Cristo. Cuando se proyectó este documental a uno de los grupos, varias participantes afirmaron identificarse con las patronas, vinculándolas a sus experiencias, tanto laborales como familiares de cuidado, atención y ayuda hacia otras personas. Cuando se entró en la discusión generada por el documental, las asistentes comenzaron a problematizar sobre el rol asignado y asumido como mujeres, en el cual se espera que ayuden, amparen, alimenten y cuiden a quienes se encuentran en condiciones de vulnerabilidad, convirtiendo sus días en jornadas de trabajo asistencial interminables. Lo anterior se discute a partir del sistema de acumulación capitalista, en el cual la labor de cuidado no es considerado trabajo por no producir bienes y servicios que generen ganancias (Carcedo, Chaves y Lexartza, 2011), a pesar de que a partir del trabajo reproductivo invisible y no pagado que realizan las mujeres, los dueños de los medios de producción obtienen grandes ganancias en el sistema capitalista (Caamaño, 2012b).

Usualmente, las funcionarias que desempeñan labores de cuido se encuentran en los "escalones inferiores del reconocimiento social y laboral" (Carcedo, Chaves y Lexartza, 2011: 28). En muchos casos, esto implica que tampoco puedan trascender sus tareas asistenciales, limitándose así su capacidad de organización para la transformación de las condiciones que afectan a la población migrante $y$ a ellas mismas, en su capacidad de incidir en políticas públicas.

Conforme se profundizan el modelo neoliberal y las políticas migratorias restrictivas en los diferentes países, se incrementa el orden patriarcal $y$ las demandas hacia las mujeres se hacen más intensas y complejas. Por ejemplo, ante la violencia desatada en México, en contra 
de la población migrante en tránsito y su desaparición, mujeres hondureñas han emprendido una serie de acciones para buscar a sus seres queridos, entre ellas, la formación de la " $\mathrm{Ca}$ ravana de Madres Centroamericanas: sigo tus pasos con la esperanza de encontrarte", la cual entre el 30 de octubre y el 14 de noviembre de 2011, recorrió diferentes sitios en México, habló con autoridades, con medios de comunicación y con todas las personas que pudieran darles indicios sobre el destino de sus seres queridos (Caamaño, 2012b).

La solidaridad presente en estas mujeres, así como en las patronas, cuestiona un sistema injusto que abandona y maltrata a los seres humanos, pero al mismo tiempo, las somete a un régimen de trabajo bajo condiciones de pobreza, mientras que los estados mexicano y centroamericanos siguen manteniendo las condiciones que vulneran a la población y que generan grandes "crisis de cuidados" (Caamaño, 2012b).

\section{CONCLUSIÓN: MIGRACIONES, SUBJETIVIDAD Y GÉNERO}

Al hacer un análisis de algunos de los contenidos discutidos en el Seminario sobre Migración y Subjetividad con grupos de mujeres, funcionarias o voluntarias en organizaciones que trabajan con población migrante, se ha constatado la imbricación de los procesos migratorios con subjetividades enmarcadas en construcciones de clase, género y nacionalidad, entre otras. Desde ese contexto, los afectos, como forma más evidente de subjetividad, marcan la relación que se establece con las personas a las cuales se intenta brindar un servicio. Los afectos surgen como testimonio de la vivencia migratoria de las personas, en un país cuya construcción simbólica ideal parte de la carencia de esta experiencia para la mayoría de la población y que tiende a la homogenización o expulsión de lo diferente.

A su vez, los afectos son un elemento que forma parte de la provisión de cuidados que se ha asignado a las mujeres en nuestra sociedad patriarcal. Así, las mujeres que atienden a la población migrante asumen el cuidado; es decir, una gran cantidad de la parte reproductiva que sostiene la labor productiva de la población migrante. Entre más desprotección genera el Estado neoliberal que restringe la regularización de la población inmigrante, más trabajo reproductivo asumen las personas migrantes y las organizaciones que las atienden.

Se pudo observar que mientras son las mujeres quienes se involucran más en la atención a las personas migrantes, siguen siendo pocas las que participan en espacios de decisión política, por lo que temas como las cadenas globales de cuido, no han sido transversales en las políticas migratorias. Así se perpetúa la división entre lo público y lo privado, establecida desde el modelo patriarcal y se sobrecarga de trabajo a las mujeres.

La naturalización de la provisión de cuidados por parte de las mujeres y la supuesta realización personal de estas al hacerlo, apelan a la subjetividad $y$ los afectos de los y las funcionarias involucradas para el mantenimiento de estas labores, lo cual resulta en escasa asignación de recursos por parte del Estado, apelando al trabajo voluntario y al papel de las instituciones religiosas. Esto inevitablemente compromete la calidad de servicios brindados, así como, el desempeño de quienes colaboran en el proceso.

Si bien, la labor asistencial en la atención de necesidades inmediatas de la población migrante es un aporte sumamente valioso por parte de las ONGs, es imprescindible que estas acciones se desarrollen de manera paralela a la lucha, con el fin de exigir al Estado, las condiciones $y$ recursos necesarios para el cumplimiento de los derechos humanos de esta población. Cualquiera de estos esfuerzos que no vaya acompañado del otro, cercenará la posibilidad de un trabajo sostenible y en mejores condiciones, tanto para quienes migran como para quienes atienden desde el ámbito profesional.

\section{BIBLIOGRAFÍA}

LIBROS

Borda, Fals. "La investigación-acción participativa: Política y epistemología". La Colombia de hoy. Bogotá: cerec, 1986.

Caamaño, Carmen. "Criando niños en Nueva York: una interpretación psicoanalítica 
sobre narcisismo, agresión y fetichismo en la relación entre dos culturas". Teorías Psicosociales. Mirta González (comp.). San José, Costa Rica. Editorial de la Universidad de Costa Rica, 2010a: 287-311.

Caamaño, Carmen. Entre "arriba" y "abajo": la experiencia transnacional de la migración de costarricense a Estados Unidos. San José, Costa Rica: Editorial de la Universidad de Costa Rica, 2010b.

Carcedo, Ana; Chaves, María José y Lexartza, Larraitz. Cadenas globales de cuidados: el papel de las migrantes nicaragüenses en la provisión de cuidados en Costa Rica. Santo Domingo: onu Mujeres, 2011.

Montaño Virreira, Sonia. "El cuidado en acción”. El cuidado en acción: entre el derecho y el trabajo. Cuadernos de la CEPAL 94. Coral Calderón y Sonia Montaño (coords.). Santiago, Chile: Naciones Unidas, 2010.

Freire, Paulo. Pedagogía del oprimido. México: Siglo xxi, 1983.

\section{REVISTAS}

Caamaño, Carmen. "La ambigüedad como salud mental: a construcción de identidades nacionales entre migrantes transnacionales costarricenses". Revista Procesos Psicológicos y Sociales 1-2 (6). México. Facultad de Psicología, Universidad Veracruzana. 2010c: 1-25.

Caamaño, Carmen. "Procesos de acumulación, migración transnacional y subjetividad en Los Santos, Costa Rica: una perspectiva de investigación desde la economía política cultural crítica". Reflexiones. Jornadas de investigación interdisciplinaria. Universidad de Costa Rica, 2011: 107-124.

Caamaño, Carmen. "Espacios de gubernamentalidad entre el "centro" y la "periferia": definiendo la migración y el desarrollo en la zona de Los Santos". Reflexiones 91 (1). Universidad de Costa Rica, 2012a: 177-190.
Matteucci, Anna. "Participación, ausencias e institucionalidad en la zona de los Santos. Un análisis con enfoque de género". Revista Feminista Casa de la Mujer 15 (20). Costa Rica. Instituto de Estudios de la Mujer, Universidad Nacional, 2011: 109-122.

\section{TEXTOS ELECTRÓNICOS}

Asamblea Legislativa de la República de Costa Rica. "Ley nro. 8764. Ley General de Migración y Extranjería de Costa Rica". La Gaceta $170.1^{\circ}$ de setiembre de 2009. Vigente desde el $1^{\circ}$ de marzo de 2010. En: $<$ http://www.migracion.go.cr/institucion/ leyes_reglamentos.html>.

OTROS

Caamaño, Carmen. "Migrantes y oNGs: la resistencia en el mundo del trabajo del cuidado en el periodo neoliberal". Ponencia. 7th Conference on Social Policy. Espirito Santo, Brasil. Junio, 2012b.

Caamaño, Carmen; Navarro, Yirlany; Matteucci, Anna y Agüero, Jazmín. "Viviendo el proceso migratorio: hacia una perspectiva crítica de las migraciones". Ponencia. VII Congreso Nacional de Psicología. San José, Costa Rica. Mayo, 2010.

Pautassi, Laura. "Nuevos desafíos para el abordaje del cuidado desde el enfoque de derechos". Ponencia. Reunión de Especialistas Futuro de las familias y Desafíos para las Políticas Públicas. Santiago, Chile: Comisión Económica para América Latina (CEPAL). Noviembre, 2007.

Prieto, Nieves y López, Fernando. El tren de las moscas [documental]. Veracruz, México: ALOKATU, 2010.

Fecha de ingreso: 13/01/2014 Fecha de aprobación: 01/04/2014 
